
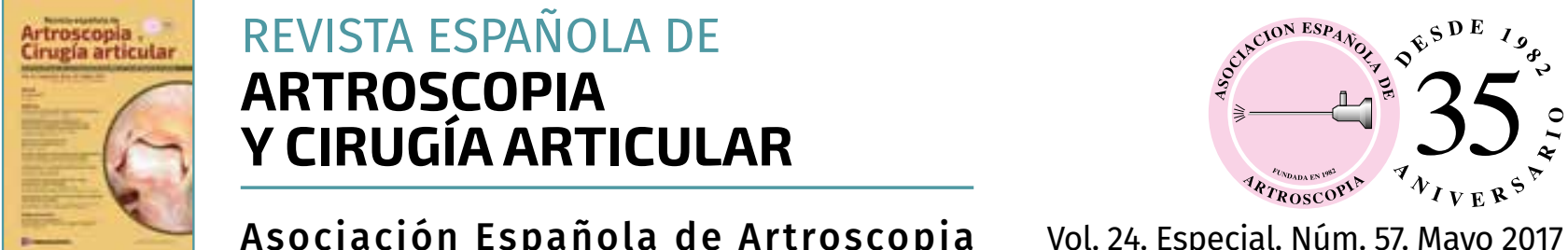

Vol. 24. Especial. Núm. 57. Mayo 2017

ISSN: 2386-3129 (impreso)

2443-9754 (online)

\title{
Original
}

\section{Epidemiología de los procedimientos artroscópicos en España. Resultados de la encuesta de actividad artroscópica de 2014}

\author{
J.L. Prieto Deza', M.A. Ruiz Ibán², M. García Navlet ${ }^{3}$, J.L. Ávila Lafuente ${ }^{4}$, \\ R. Cuéllar Gutiérrez ${ }^{5}$ A. Calvo Díaz
}

${ }^{1}$ Servicio de Traumatología y Cirugía Ortopédica. Hospital Mateu Orfila. Menorca, Islas Baleares

2 Unidad de Hombro y Codo. Hospital Universitario Ramón y Cajal. Madrid

${ }^{3}$ Unidad de Patología de Hombro y Codo. Hospital ASEPEYO. Coslada, Madrid

${ }^{4}$ Unidad de Cirugía de Miembro Superior. Hospital de la Mutua MAZ. Zaragoza

${ }^{5}$ Hospital Universitario Donostia. Policlínica Gipuzkoa QuirónSalud. San Sebastián

${ }^{6}$ Servicios Médicos de Arthrosport. Zaragoza

Correspondencia:

Dr. José Luis Prieto Deza

Correo electrónico: jlprietod@hotmail.com
Recibido el 27 de febrero de 2017

Aceptado el 22 de marzo de 2017

Disponible en Internet: mayo 2017

\section{RESUMEN}

Objetivo: el objetivo de este estudio fue realizar una encuesta de la actividad artroscópica en el territorio español para el año 2014.

Material y metodologia: se obtuvo información estadística a través de una plataforma informática de los diferentes centros hospitalarios españoles, públicos y privados, donde se realizaba dicha actividad. Los datos estaban basados en un perfil general, otro referente a la actividad y un tercero referido a datos concretos de procedimientos artroscópicos por articulación. Se registraron 59.897 artroscopias que suponían un $30,8 \%$ de la muestra posible, lo que supone un importante incremento de actividad artroscópica en relación con estudios previos.

Resultados: las articulaciones cuantitativamente más intervenidas fueron la rodilla y el hombro por ese orden, con un elevado protagonismo de los procedimientos en cirugía mayor ambulatoria con igual presencia en centros públicos y privados. Se detectó una aparición emergente de otras articulaciones como cadera, tobillo y mano-mu-

\begin{abstract}
Epidemiology of arthroscopic procedures in Spain. Results of the 2014 arthroscope activity survey
\end{abstract}

Objective: we perform a survey of arthroscopic activity in the Spanish territory for the year 2014.

Material and methodology: statistical information was obtained through a computer platform of the different Spanish public and private hospitals where this activity was carried out. The data were based on a general profile, another on activity and a third on specific data on arthroscopic procedures by articulation. A total of 59,897 recorded arthroscopies were obtained, accounting for $30.8 \%$ of the possible sample. Which represents an important increase of arthroscopic activity in relation to previous studies.

Results: the quantitatively most important joints were the knee and shoulder in that order, with a high profile of procedures in ambulatory major surgery with equal presence in public and private centers. An emergent appearance of other joints such as hip, ankle and hand-wrist was detected

https://doi.org/10.24129/j.reaca.24e57.fs1702007

FS $\odot 2017$ Fundación Española de Artroscopia. Publicado por Imaidea Interactiva en FONDOSCIENCE ${ }^{\circledR}$ (www.fondoscience.com) Este es un artículo Open Access bajo la licencia CC BY-NC-ND (www.creativecommons.org/licenses/by-nc-nd/4.0/). 
ñeca, con predominio en los centros privados sobre los públicos.

Conclusión: se constata un aumento del número y la complejidad de este tipo de procedimientos con respecto a publicaciones previas y que este tipo de estudios son útiles para conseguir una mejora en referencia a la actividad artroscópica.

Palabras clave: Encuesta. Cuestionario. Artroscopia. Cirugía. Rodilla. Hombro.

\section{Introducción}

La cirugía ortopédica ha experimentado avances significativos durante la segunda mitad del siglo veinte. Mientras que en los años sesenta y setenta se produjo una popularización de los procedimientos de sustitución articular, el perfeccionamiento de los instrumentos disponibles para realizar procedimientos con control artroscópico supuso una revolución en el manejo de las lesiones de rodilla primero, en el hombro después y eventualmente en todas las articulaciones de la economía del sistema musculoesquelético(1).

El verdadero impacto que han tenido las técnicas artroscópicas en la salud de la población es evidente pero difícil de estimar. Identificar el número real de procedimientos artroscópicos que se realizan tiene importancia pues da información que debería tener impacto en la política sanitaria y en los recursos destinados a la formación en dichas técnicas. Además, es un paso necesario previo al planteamiento de la realización de registros nacionales de procedimientos específicos; estos registros se han demostrado como herramientas de alta utilidad para evaluar los resultados de dichos procedimientos y optimizar los resultados de estos.

La Asociación Española de Artroscopia (AEA) agrupa a casi 1.000 traumatólogos españoles que tienen un interés especial en estas técnicas. Desde su comienzo, la AEA ha tenido una vocación principalmente docente e investigadora, pero también se considera abanderada de la defensa de la utilidad de estas técnicas en el manejo de los problemas articulares de la población. En esa línea, se ha considerado prioritaria la cuantificación real del papel que tienen estas técnicas en España. Un primer esfuerzo en esa línea cristalizó en la rea- predominating here activity in the private centers over the public.

Conclusion: we observed an increase in the number and complexity of this type of procedure with respect to previous publications and that this type of studies are useful to achieve an improvement in reference to the arthroscopic activity.

Key words: Survey. Questionnaire. Arthroscopy. Surgery. Knee. Shoulder.

lización del "Informe sobre el perfil de la cirugía artroscópica en España"(2), basado en un cuestionario entregado en el año 2000 a un grupo de 240 miembros de la sociedad. Este intento de cuantificación de la actividad artroscópica tiene su reflejo fuera de nuestras fronteras en estudios realizados en los EE. UU. El objetivo de la Encuesta de Actividad Artroscópica en España (2014) es identificar el número de procedimientos artroscópicos realizados en España en el año 2014, conocer sus características principales, así como evaluar algunos aspectos relacionados con la docencia en cirugía artroscópica y el papel que distintas empresas que suministran material para cirugía artroscópica en el mercado español. En este artículo se presentan exclusivamente los datos relacionados con el número y las características de los procedimientos artroscópicos realizados en España en 2014.

\section{Material y métodos}

La Junta Directiva de la AEA asignó el diseño y el desarrollo de la encuesta al vocal de Estudios de dicha Junta (JLPD y JLAL) que, a su vez, escogió un comité científico (formado por JLPD, JLA y MGN) responsable del diseño de la encuesta y la definición del objeto de la misma. Para el desarrollo técnico de la encuesta se seleccionó, previo concurso público, a la empresa Torres Pardo. La población a la que se dirigió la encuesta era la de la totalidad de los cirujanos ortopédicos españoles que realizan procedimientos quirúrgicos con asistencia artroscópica. Para acceder a esta población, de difícil identificación, se decidió focalizar la atención en los centros médicos donde se realizan procedimientos quirúrgicos: se extrajo el listado de los 


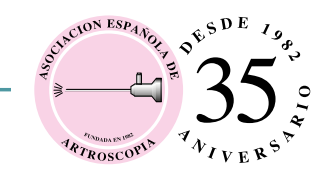

centros médicos del Catálogo Nacional de Hospitales del Ministerio de Sanidad, Servicios Sociales e Igualdad de $2014^{(3)}$. De esa lista se excluyeron manualmente aquellos en los que es poco probable por su finalidad asistencial que realicen artroscopias (monográficos no traumatológicos, maternales, geriátricos, psiquiátricos, de rehabilitación, etc.), quedando un total de 364 centros.

El objeto de la encuesta fue recabar detalles del mayor número posible de procedimientos artroscópicos realizados en España durante el año natural 2014. La recogida de datos se extendió durante el primer cuatrimestre de 2015.

Para la recogida de datos se diseñó una encuesta para ser rellenada on-line. Los campos de la encuesta incluian detalles sobre: la persona que rellenaba la encuesta, las características del centro, el número de procedimientos por cada articulación y sus características, así como aspectos relacionados con las empresas suministradoras de los productos para cirugía artroscópica y la docencia de las técnicas artroscópicas. En el Anexo 1 se muestra un listado completo de todas las variables recogidas. En el Anexo 2 se muestran las capturas de pantalla de un ejemplo de encuesta rellena.

Se contactó mediante correo electrónico o correo estándar dirigido al responsable médico o al jefe del Servicio de Traumatología y Cirugía Ortopédica de cada centro. En este se les solicitaba que rellenasen la encuesta on-line o, en su defecto, que indicasen a alguien de su equipo que lo hiciese por ellos. Suplementariamente en el tercer congreso conjunto de la AEA-SEROD (Sociedad Española de la Rodilla) (realizado en Madrid del 22 al 24 de abril de 2015), se incluyó en la carpeta del congreso una copia de dicha carta, instando al congresista a rellenar la encuesta si nadie de su institución lo había hecho. Los resultados de todas las encuestas recogidas fueron incorporados a una base de datos informatizada. De esta base de datos, una vez normalizados y uniformizados los datos, se extrajeron los resultados.

Se obtuvieron un total de 146 respuestas a la encuesta. En la primera fase de solicitud on-line se enviaron notificaciones a 364 centros, obteniéndose 112 respuestas (30,8\%). La distribución por comunidades autónomas se presenta en la Figura 1. Durante la segunda fase de reclutamiento, durante el congreso celebrado en Madrid, se obtuvieron 34 respuestas más, 4 de ellas correspondientes a centros que no habían sido inclui-

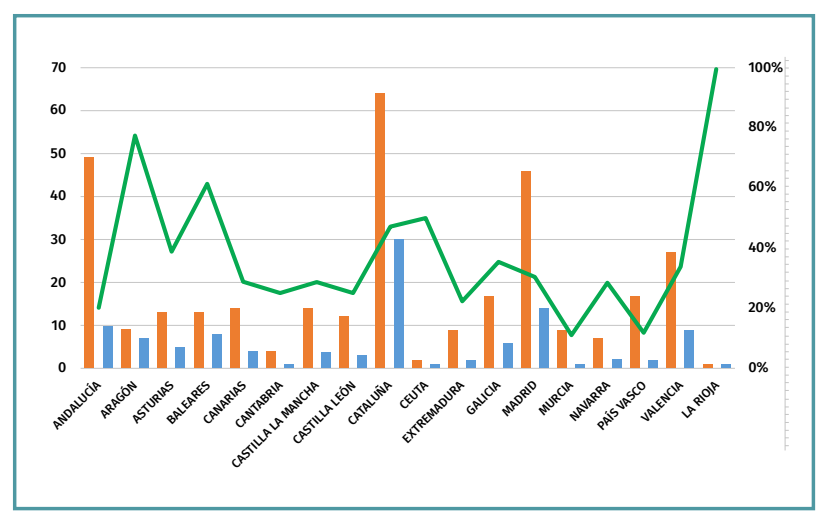

Figura 1. Número total de centros consultados (columnas naranjas) y de centros que respondieron (columnas azules) en cada comunidad autónoma (referido al eje de ordenadas izquierdo). Porcentaje de centros (línea verde) que respondieron en cada comunidad autónoma (referido al eje de ordenadas derecho).

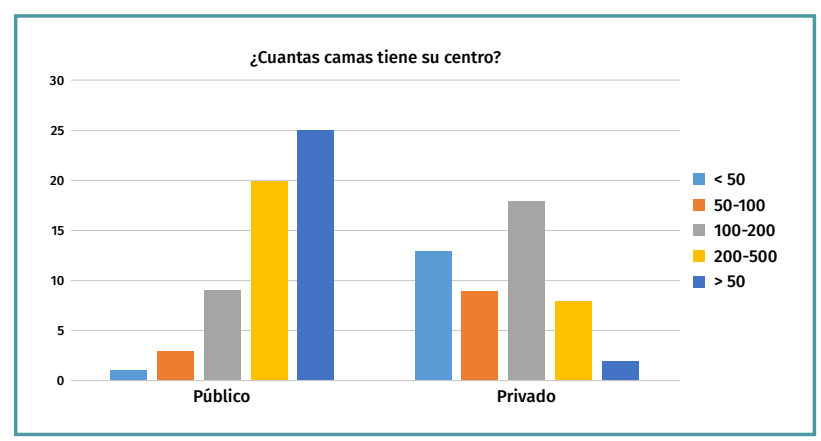

Figura 2. Número de camas que tiene el centro.

dos en el listado inicial, de cirujanos o equipos de cirujanos que mantenían actividad en distintos centros quirúrgicos y que respondieron unificando sus resultados.

Respecto a los profesionales sanitarios que respondieron a la encuesta, un $61 \%$ fueron jefes de servicio, el $12,7 \%$ médicos adjuntos, el $10 \%$ jefes de la Unidad de Artroscopia, el 6,3\% jefes de departamento, el 7\% traumatólogos independientes, el $2 \%$ directores médicos y el $1 \%$ otros, con un tiempo medio de experiencia de 27 años.

El 55,2\% de los centros que respondieron la encuesta eran públicos, entendiéndose como tal que pertenecen al Servicio Público de Salud, y el resto $(44,8 \%)$ se distribuían entre centros privados, mutuas, concertados o grupos de trabajo de carácter privado. El número de camas de los centros puede apreciarse en la Figura 2 y el porcen- 


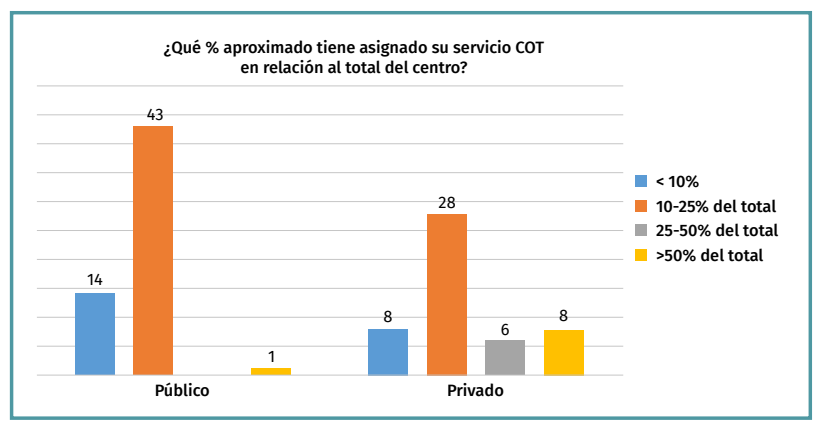

Figura 3. Porcentaje de camas asignadas en el centro al Servicio de Cirugía Ortopédica y Traumatología (COT).

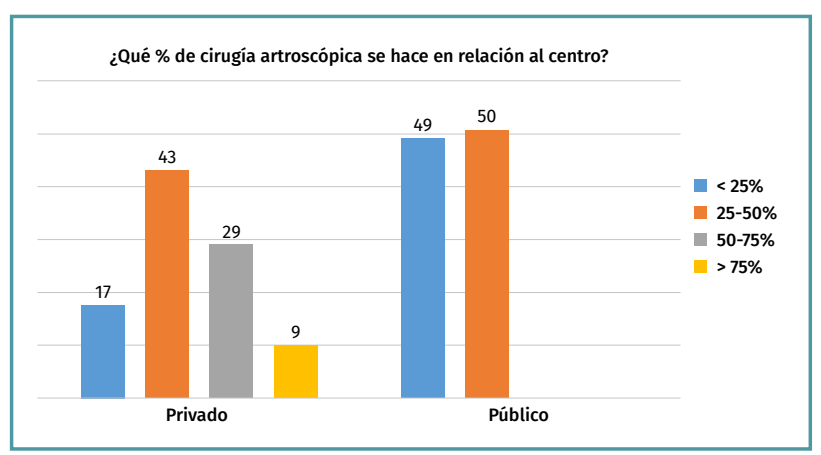

Figura 4. Porcentaje de actividad de los centros que corresponde a cirugía artroscópica.

taje de camas asignadas al Servicio de Traumatología en la Figura 3.

En el 55,53\% de los centros con menos de 200 camas, más del $75 \%$ de los especialistas del servicio realizaban artroscopias en su práctica diaria. En los centros con un número de camas entre 200 y 500 , en el $19,05 \%$ de los especialistas las
Tabla 2. Media de artroscopias anuales según el centro

\begin{tabular}{l|c|c|c}
\hline Articulación & Centros privados & Centros públicos & Total \\
\hline Rodilla & 293 & 257 & 272 \\
\hline Hombro & 119 & 117 & 118 \\
\hline Tobillo & 32 & 15 & 23 \\
\hline Mano & 22 & 18 & 20 \\
\hline Cadera & 24 & 14 & 18 \\
\hline Codo & 12 & 5 & 8 \\
\hline Raquis & 3 & 2 & 2 \\
\hline
\end{tabular}

artroscopias ocupaban entre el 50 y el $75 \%$ de su actividad diaria, pasando al $18,75 \%$ si supone más del 75\% de su actividad quirúrgica habitual. En cambio, en hospitales de más de 500 camas, solo el 7,32\% de los facultativos realizaron cirugías artroscópicas en más del 75\% de su actividad (Figura 4).

Se registraron un total de 59.897 artroscopias en 146 centros; la distribución por articulaciones y el número absoluto se muestra en la Tabla 1 y la media de artroscopias según el tipo de centro en la Tabla 2.

\section{Manejo y proceso de datos}

Los formularios fueron enviados en línea a través de un área privada a la que solo se accede mediante usuario y contraseña. Los formularios tenían reglas de validación y listas predefinidas de opciones de selección, para así garantizar la calidad óptima de los datos enviados. Cada usuario tenía la capacidad/posibilidad de acceder a su área privada y actualizar el formulario hasta que la versión final quedaba confirmada para envío. A partir de ese momento, el formulario quedaba bloqueado.

Los datos se almacenaron en una base de 


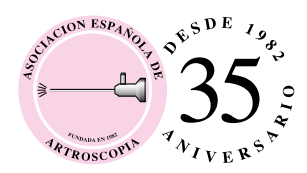

datos relacional, lo que permite gestionarla fácilmente, consultar y filtrar según diferentes criterios. Para el informe solo se consideraron formularios confirmados. Los formularios parcialmente completados fueron descartados. Todos los formularios confirmados fueron revisados, para detectar posibles inconsistencias, información incompleta o datos sesgados. Las entradas duplicadas fueron eliminadas, con respecto al número de artroscopias recogidas en cada centro.

Los formularios enviados y confirmados fueron exportados en formato Excel y gestionados en hojas de datos separadas para cada área analizada. Los datos se analizaron teniendo siempre en cuenta el tipo de centro y la provincia del mismo. Para los campos multiopción donde se seleccionó un intervalo, se ha considerado el valor medio del mismo.

Los valores promedio y medio de desviación se calcularon en la hoja de Excel. Cada tipo de artroscopia se estudió por separado.

\section{Resultados}

Se realizaron 33.736 cirugías artroscópicas de rodilla en 146 centros. Su distribución por patología se muestra en la Figura 5. Se interrogó sobre el porcentaje de procedimientos que se realizaban en forma de cirugía mayor ambulatoria (CMA): el 86\% de las cirugías meniscales y el $64 \%$ de las cirugías de cartílago, reduciéndose este porcentaje al 34\% de la cirugía de ligamento cruzado anterior (LCA) y al $2 \%$ de las cirugías de ligamento cruzado posterior (LCP). El 55\% de los centros realizaban suturas meniscales, porcentaje que subía al 59\% en los centros públicos. Respecto al tipo de fijación para las plastias de reconstrucción de LCA, la fijación femoral fue mayoritariamente de tenosuspensión (52\%), seguida de la transfixiante (26\%) y con tornillo interferencial (17\%); en la tibia, la fijación fue con tornillos interferenciales en la amplia mayoría (80\%), seguido de un $13 \%$ de fijaciones transfixiantes.

Se realizaron artroscopias de hombro en los 146 centros registrados. La distribución por patología de las 16.608 cirugías artroscópicas de hombro registradas se presenta en la Figura 6 . Se interrogó también sobre el porcentaje de procedimientos que se realizaban en forma de CMA. El $66 \%$ de las cirugías artroscópicas de hombro correspondieron al espacio subacromial, el 54\% fue-

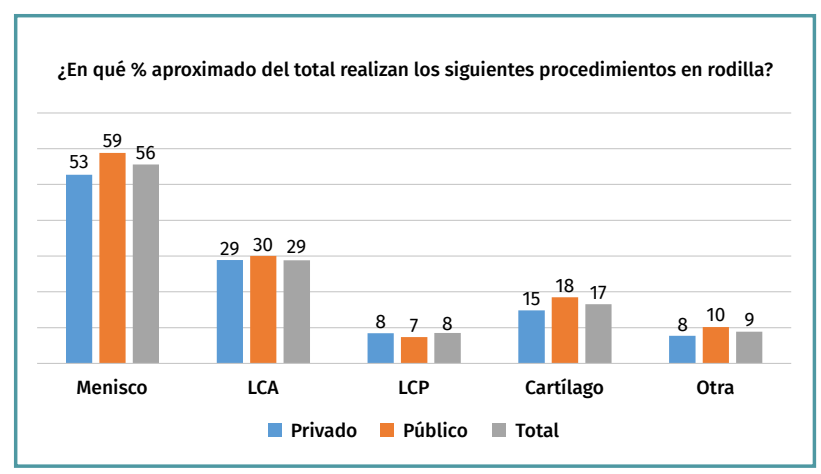

Figura 5. Distribución del tipo de procedimientos en la artroscopia de rodilla.

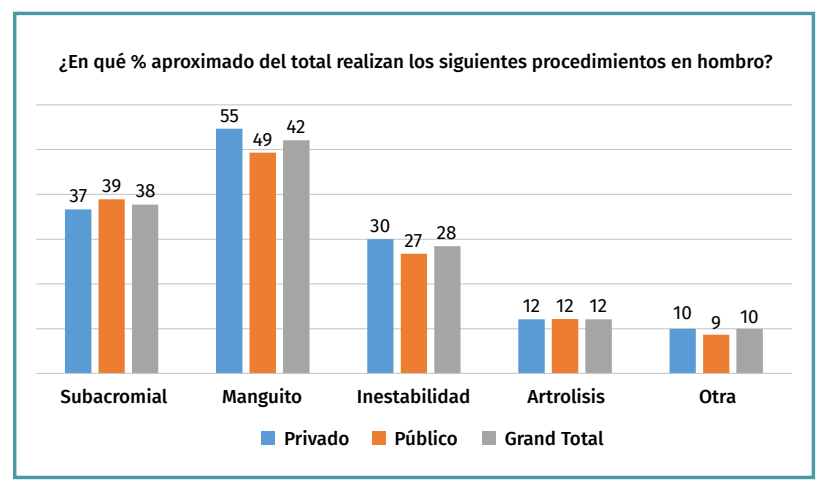

Figura 6. Distribución del tipo de procedimientos en la artroscopia de hombro.

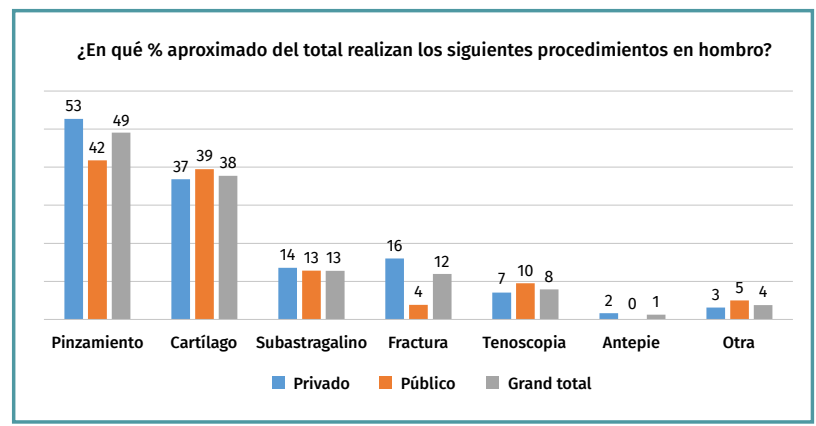

Figura 7. Distribución del tipo de procedimientos en la artroscopia de tobillo.

ron reparaciones de manguito de los rotadores y el $55 \%$ a cirugías de inestabilidad. Se utilizaron $3,25 \pm 0,63$ implantes en las cirugías de inestabilidad y 2,64 $\pm 1,02$ implantes en las cirugías de reparación de manguito rotador.

Se realizaron 3.113 artroscopias de tobillo en 139 de los 146 centros registrados (95,20\%) (Figura 7). 


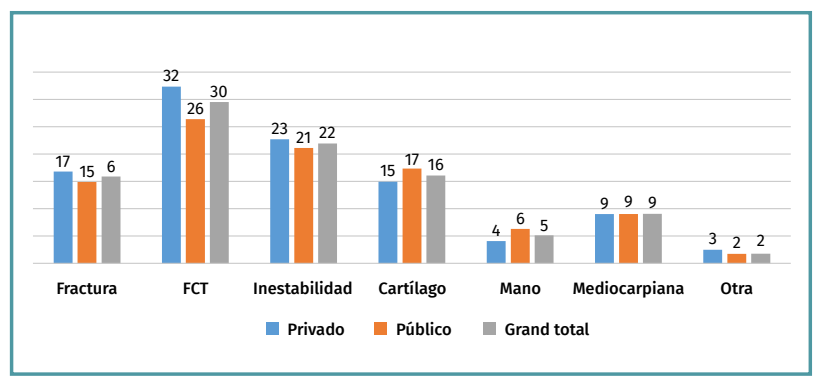

Figura 8. Distribución del tipo de procedimientos en la artroscopia de mano/muñeca.

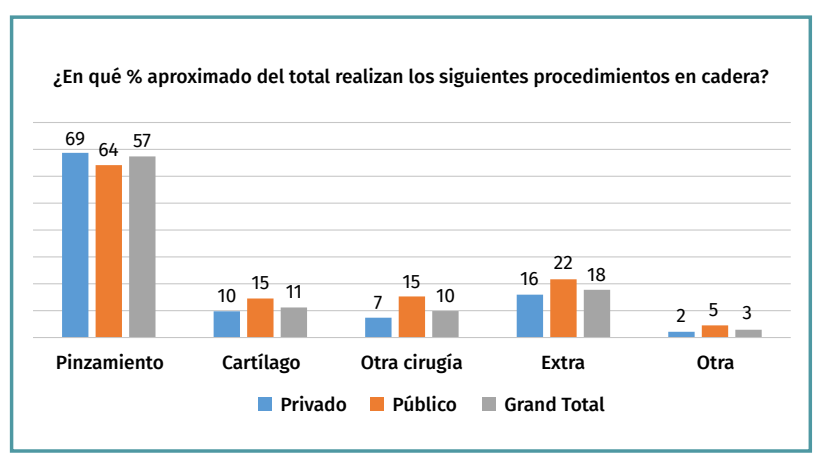

Figura 9. Distribución del tipo de procedimientos en la artroscopia de cadera.

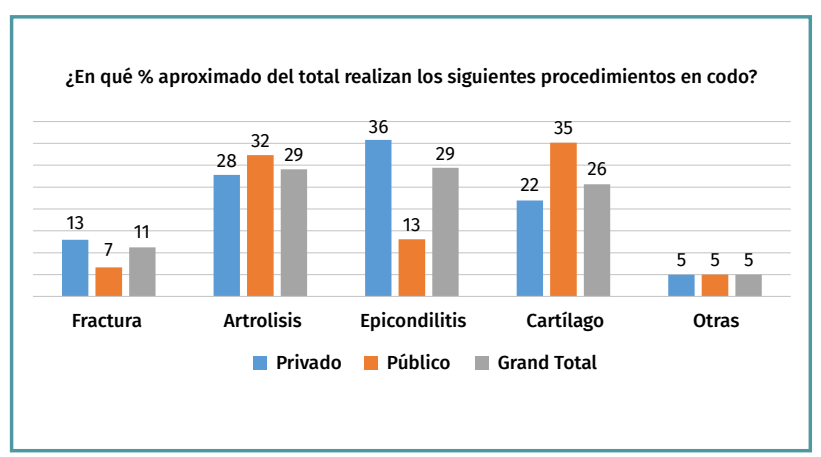

Figura 10. Distribución del tipo de procedimientos en la artroscopia de codo.

Las 2.615 cirugías artroscópicas de mano y de muñeca contabilizadas se ofertaron en 121 de los 146 centros registrados (82,87\%) (Figura 8). Se realizaron 2.427 cirugías artroscópicas de cadera en 116 de los 146 centros registrados (79,45\%) (Figura 9).

Las 1.113 cirugías artroscópicas de codo registradas se realizaron en 117 de los 146 centros registrados (80,01\%) (Figura 10). Se efectuaron 285 artroscopias de raquis, en 51 de los 146 centros registrados (34,93\%). El 75\% de los procedimientos correspondieron a la cirugía de hernia discal.

Se preguntó sobre la realización de artroscopias de revisión en cada centro. Fueron habituales las artroscopias de revisión de la rodilla en el $94 \%$ de los centros, de hombro en el $91 \%$, de tobillo en el $43 \%$, de muñeca en el $37 \%$ y de codo en el $26 \%$. Se observó que las revisiones artroscópicas de cirugías previas se hacían más en centros con mayor volumen en esa articulación.

\section{Discusión}

La principal conclusión de este estudio es que el uso de los procedimientos artroscópicos en cirugía ortopédica se ha generalizado en la mayor parte de los centros. Cada vez se realizan más artroscopias, por más cirujanos y sobre más articulaciones.

Las comparaciones de este estudio deben de hacerse en primer lugar con la encuesta realizada por la Junta Directiva de la AEA durante el año $2000^{(2)}$. En esta se recogieron datos de 240 centros, obtenidos de 190 cirujanos miembros de la AEA (el 53\% de los miembros de entonces), en los que se realizaron 45.316 procedimientos artroscópicos. Es por tanto una encuesta con unas características, y limitaciones, muy similares a la presentada aquí.

Respecto al número total de artroscopias por centro, los datos respecto al año 2000 son claramente diferentes: de 188 artroscopias por año de media en cada centro en 2000 , se pasa a 410 artroscopias por centro en 2014, lo que presupone un incremento, esperado, del número de artroscopias totales, algo en sintonía con otros estudios que confirman que el número de procedimientos artroscópicos está en claro aumento en todas las articulaciones ${ }^{(4-7)}$.

Por otro lado, el perfil de articulaciones intervenidas ha cambiado. En relación con el estudio de 2000, el porcentaje de artroscopias de rodilla baja, del 78 al 56\%, no porque disminuya su número sino porque suben porcentualmente las artroscopias de las demás articulaciones. El hombro pasa del 17 al 27,73\%, el tobillo del 3 al $5,20 \%$, la cadera del 0,3 al 4,05\% y la artroscopia de mano y muñeca del 1 al 4,37\%.

El número de procedimientos artroscópicos de rodilla hechos en cada centro ha variado en- 


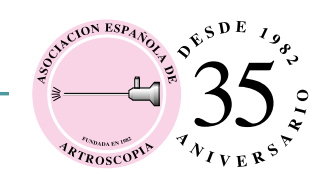

tre ambas encuestas, 145 anuales por centro en $2000^{(2)}$ y 272 anuales por centro en 2014. Este aumento en el número total de intervenciones va acompañado de otros hechos relevantes como el alto número de intervenciones totales realizadas de forma ambulante, una tendencia que es bien patente en los EE. UU. tanto a nivel general ${ }^{(8)} \mathrm{como}$ en cirugía de reconstrucción de LCA ${ }^{(6)}$. Sorprende que en 14 años no haya cambiado significativamente el número de centros donde se realiza sutura meniscal (del 60\% en 2000 al 57\% en 2014), una tendencia opuesta a la de los EE. UU., donde hay un fuerte incremento de la tasa de suturas meniscales ${ }^{(7)}$. Por otro lado, el incremento del número de reconstrucciones de LCA realizadas es bien patente, en 2000 suponían solo el 22\% de las cirugías de rodilla y en 2014 suponen aproximadamente el $30 \%$ de los procedimientos, en línea con lo publicado en la bibliografía internacional ${ }^{(9)}$. En cuanto al tipo de plastia empleada en la reconstrucción del LCA, la preferencia actual en nuestra encuesta son injertos de los tendones isquiotibiales, habida cuenta del probable bajo porcentaje de injerto hueso-tendón-hueso en técnica suspensoria.

La cirugía artroscópica de hombro es una de las principales responsables de la explosión en el número de artroscopias realizadas en estos centros españoles, pues se ha pasado de 33 anuales por centro en 2000, a 118 anuales por centro en 2014. Este incremento se ve justificado por la tendencia internacional a la generalización en el uso de técnicas artroscópicas para la reparación del manguito rotador ${ }^{(10)}$ y de la inestabilidad de hombro(11). Estas indicaciones pasaron de suponer el 8 y el $14 \%$ de la patología intervenida en 2000 al 50 y el $30 \%$ en 2014, respectivamente. La tendencia mundial al aumento de la realización de estos procedimientos en $\mathrm{CMA}^{(12)}$ se aprecia en esta encuesta, realizándose el 50\% de los procedimientos con este esquema.

En cirugía artroscópica de tobillo no se ha producido un incremento lineal como también se aprecia en otros países ${ }^{(4)}$. Se ha pasado de 6 artroscopias anuales en 2000 a 23 en 2014 y el porcentaje de centros donde se realizan ha pasado del 72 al 95\%.

La cirugía artroscópica de mano y muñeca ha tenido también un incremento significativo, de 2 a 20 procedimientos por centro encuestado. Este incremento probablemente es debido a la gene- ralización del uso de esta técnica por parte de los cirujanos de mano, que llega hasta el $50 \%{ }^{(13)}$. Este es un procedimiento que se realiza de forma ambulatoria ${ }^{(12)}$.

La artroscopia de cadera ha irrumpido también con fuerza en estos 14 años: realizada anecdóticamente por solo 1 de cada 6 centros en el 2000 (a razón de menos de 1 artroscopia anual en el cómputo total), llega en la encuesta actual a 18 procedimientos anuales. Esta tendencia se ha visto corroborada recientemente por el análisis del número de procedimientos de este tipo efectuados en el País Vasco(5).

La hermana pobre de la cirugía artroscópica sigue siendo la artroscopia de raquis. Se realizan, en los centros encuestados, apenas 300 casos anuales.

Este estudio presenta algunas limitaciones metodológicas. El bajo porcentaje de respuestas -han respondido menos del $40 \%$ de los centros encuestados- y la no realización de una estratificación de las respuestas escogidas por el tipo de hospital y su tamaño hacen imposible inferir, aunque fuese de manera imprecisa, el número real de procedimientos artroscópicos realizados en España. La encuesta del año 2000 lo hizo con datos similares, pero con una participación mucho mayor de los miembros de la AEA (> 50\%) y teniendo en cuenta que en aquellos momentos la realización de estos procedimientos estaba mucho más centralizada en un menor número de cirujanos. Otra limitación respecto a la cuantificación de los procedimientos artroscópicos realizados es que, durante la encuesta, se ofrecía la posibilidad de informar sobre el número de artroscopias en formato semicuantitativo, esto es, indicaban un número aproximado, "se realizaron 5-10 artroscopias de cadera en el 2014", frente al número exacto; esta limitación es solo parcial, ya que, perdida la posibilidad de estimar realmente el número total de procedimientos en el territorio nacional, la obtención de la información relativa a la distribución por articulaciones y por el tipo de procedimiento no se ve tan sesgada por este problema de recogida de datos.

Las técnicas artroscópicas suponen una cirugía relevante en la práctica quirúrgica de los cirujanos encuestados. Se realizan múltiples procedimientos por cada vez un mayor número de cirujanos. Desde el año 2000, la cirugía artroscópica de rodilla ha aumentado significativamente 
su complejidad, mientras que la del resto de las articulaciones ha aumentado en número. Son de especial relevancia el aumento en el número de artroscopias de hombro, mano-muñeca y cadera.

\section{Bibliografía}

1. Jackson RW. A history of arthroscopy. Arthroscopy. 2010;26:91-103.

2. Junta Directiva de la Asociación Española de Artroscopia. Informe sobre el perfil de la cirugía artroscópica en España. Cuadernos de Artroscopia. 2001;8:7.

3. Catálogo Nacional de Hospitales 2014. Madrid: Ministerio de Sanidad, Servicios Sociales e Igualdad; 2014.

4. Best MJ, Buller LT, Miranda A. United States National Trends in Ankle Arthroscopy: Analysis of the National Survey of Ambulatory Surgery and National Hospital Discharge Survey. Foot Ankle Spec. 2014;8:266-72.

5. Cuéllar Ayestarán A, Cuéllar Gutiérrez R, Etxebarría-Foronda I, Sánchez Sobrino A, Ruiz Ibán MA. Evolución de los procedimientos artroscópicos de cadera en el País Vasco entre 2008 y 2013. Rev Esp Artrosc Cir Articul. 2015;22:6

6. Mall NA, Chalmers PN, Moric M, Tanaka MJ, Cole BJ, Bach BR Jr, Paletta GA Jr. Incidence and trends of anterior cruciate ligament reconstruction in the United States. Am J Sports Med. 2014;42:2363-70.
7. Parker BR, Hurwitz S, Spang J, Creighton R, Kamath G. Surgical Trends in the Treatment of Meniscal Tears: Analysis of Data From the American Board of Orthopaedic Surgery Certification Examination Database. Am J Sports Med. 2016;44:1717-23.

8. Kim S, Bosque J, Meehan JP, Jamali A, Marder R. Increase in outpatient knee arthroscopy in the United States: a comparison of National Surveys of Ambulatory Surgery, 1996 and 2006. J Bone Joint Surg (Am). 2011;93-A:994-1000.

9. Leathers MP, Merz A, Wong J, Scott T, Wang JC, Hame SL. Trends and Demographics in Anterior Cruciate Ligament Reconstruction in the United States. J Knee Surg. 2015;28:390-4.

10. Zhang AL, Montgomery SR, Ngo SS, Hame SL, Wang JC, Gamradt SC. Analysis of rotator cuff repair trends in a large private insurance population. Arthroscopy. 2013;29:623-9.

11. Malhotra A, Freudmann MS, Hay SM. Management of traumatic anterior shoulder dislocation in the 17- to 25-year age group: a dramatic evolution of practice. J Shoulder Elbow Surg. 2012;21:545-53.

12. Jain NB, Higgins LD, Losina E, Collins J, Blazar PE, Katz JN. Epidemiology of musculoskeletal upper extremity ambulatory surgery in the United States. BMC Musculoskelet Disord. 2014;15:4.

13. Obdeijn MC, Tuijthof GJ, van der Horst CM, Mathoulin C, Liverneaux P. Trends in wrist arthroscopy. J Wrist Surg. 2013;2:239-46. 


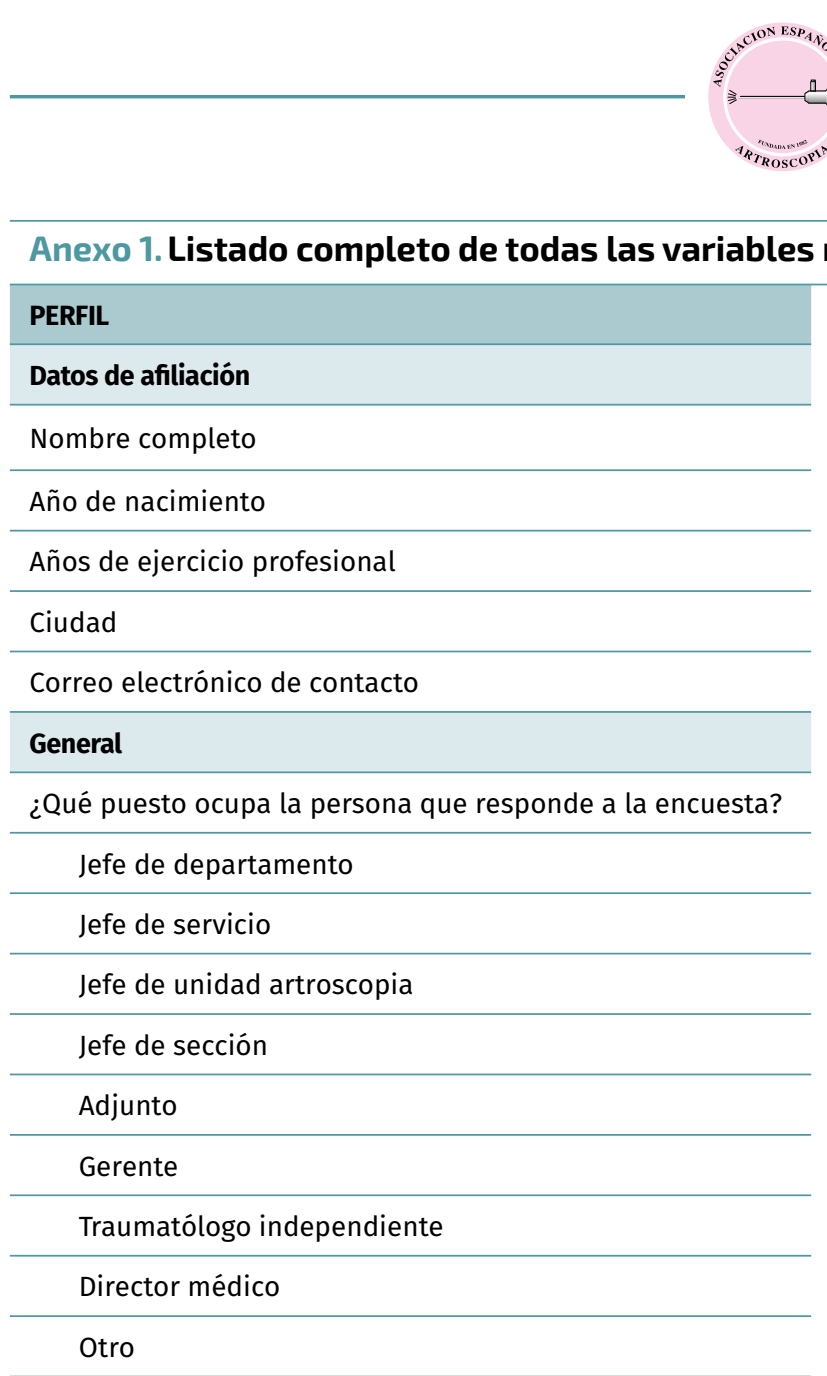

Conocía la intención de la AEA de generar una encuesta nacional de la actividad artroscópica?

¿Considera útil e interesante este tipo de estudio/encuesta?

¿Le gustaría incluir a su hospital/unidad dentro de los Centros Colaboradores y realizar la encuesta y así acceder a la información que se genere?

¿Es Ud. miembro de la AEA?

\section{CENTRO}

Sobre el Centro

¿Qué tipo de centro es?

Público

Privado

Mixto

Concertado

Mutua-Accidentes de trabajo

Como grupo privado en varios centros

Otro

¿Cuántas camas tiene su centro?

¿Qué \% aproximado de camas tiene asignado su servicio de COT en relación al total del centro?

\section{Sobre la composición del equipo}

¿Cuántos adjuntos de COT hay en el servicio?

¿Cuántos adjuntos del servicio realizan de forma habitual artroscopia?

\section{Sobre la actividad}

En el centro se hace cirugía artroscópica:

En caso afirmativo, la artroscopia está integrada en sus respectivas unidades funcionales (por ejemplo: unidad de patología de rodilla, miembro inferior, etc.)

¿Qué \% aproximado de cirugía artroscópica se hace en relación al total?

\section{Sobre la formación}

En su centro hay:

Residentes propios

Residentes rotantes

Traumatólogos visitantes nacionales

Traumatólogos visitantes internacionales

Fellows

Otro tipo de visitante o becario

No hay ningún tipo de formación

¿Cuál o cuáles de estas vías son utilizadas en su centro para la formación de los residentes y adjuntos jóvenes en el campo de la artroscopia?

En el propio centro

En estancias en centros externos nacionales

En estancias en centros externos internacionales

Cursos de formación en artroscopia propios de la AEA o avalados por la AEA

Cursos de formación en artroscopia de las casas comerciales

Cursos de formación en artroscopia de otras sociedades científicas

¿Estaría su centro dispuesto a acreditarse como Centro de Formación Artroscópica?

¿Estaría su centro capacitado para recibir a fellows nacionales o internacionales?

¿Qué papel atribuye a la AEA en la formación de los residentes y adjuntos jóvenes? 


\begin{tabular}{|c|c|}
\hline ARTROSCOPIA & ARTROSCOPIA \\
\hline Rodilla & Hombro \\
\hline $\begin{array}{l}\text { ¿Cuántas artroscopias se realizan en su centro/unidad } \\
\text { anualmente? }\end{array}$ & $\begin{array}{l}\text { ¿Cuántas artroscopias se realizan en su centro/unidad } \\
\text { anualmente? }\end{array}$ \\
\hline ¿Se realizan artroscopias de revisión? & ¿Se realizan artroscopias de revisión? \\
\hline $\begin{array}{l}\text { ¿En qué \% aproximado del total realizan los siguientes } \\
\text { procedimientos? }\end{array}$ & $\begin{array}{l}\text { ¿En qué \% aproximado del total realizan los siguientes } \\
\text { procedimientos? }\end{array}$ \\
\hline Meniscectomías y sutura meniscal & Subacromial \\
\hline LCA & Manguito \\
\hline LCP/complex & Inestabilidad \\
\hline Cartílago F-T y rotuliano & Artrolisis \\
\hline Otras & Otras \\
\hline ¿Qué tipo de fijación usa en \% aproximado? & ¿Cúantos anclajes ponen de media en inestabilidad? \\
\hline Transfixiante fémur & ¿Cuántos anclajes pone de media en manguito? \\
\hline Suspensorio fémur & ¿Con qué casas comerciales trabaja habitualmente (marque \\
\hline IF fémur & $\lim 2)$ ? \\
\hline Otro fémur & $\mathrm{S} \& \mathrm{~N}$ \\
\hline Transfixiante tibia & Arthrex \\
\hline Suspensorio tibia & Mitek \\
\hline IF tibia & Linvatec \\
\hline Otro tibia & Biomet \\
\hline ¿Hacen habitualmente sutura meniscal? & Stryker \\
\hline ¿Con qué casas comerciales trabaja habitualmente (marque & Otras \\
\hline máximo 2)? & Tobillo y pie \\
\hline$S \& N$ & ¿Cuántas artroscopias se realizan en su centro/unidad \\
\hline Arthrex & anuatmente? \\
\hline Mitek & ¿Se realizan artroscopias de revisión? \\
\hline Linvatec & $\begin{array}{l}\text { ¿En qué \% aproximado del total realizan los siguientes } \\
\text { procedimientos? }\end{array}$ \\
\hline Biomet & Pinzamiento \\
\hline Stryker & Subastragalina \\
\hline Otras & Cartilago \\
\hline & Fractura \\
\hline & Tenoscopia \\
\hline & Antepie \\
\hline & Otra \\
\hline
\end{tabular}




\begin{tabular}{|c|c|}
\hline \multicolumn{2}{|c|}{ Anexo 1. Listado completo de todas las variables recogidas en la encuesta (III) } \\
\hline ARTROSCOPIA & ARTROSCOPIA \\
\hline Tobillo y pie & Mano-muñeca \\
\hline $\begin{array}{l}\text { ¿Con qué casas comerciales trabaja habitualmente (marque } \\
\text { máximo 2)? }\end{array}$ & $\begin{array}{l}\text { ¿Cuántas artroscopias se realizan en su centro/unidad } \\
\text { anualmente? }\end{array}$ \\
\hline S\&N & ¿Se realizan artroscopias de revisión? \\
\hline Arthrex & \multirow{2}{*}{$\begin{array}{l}\text { ¿En qué \% aproximado del total realizan los siguientes } \\
\text { procedimientos? }\end{array}$} \\
\hline Mitek & \\
\hline Linvatec & Fractura \\
\hline Biomet & FCT \\
\hline Strvker & Inestabilidad \\
\hline & Cartílago/C. libres \\
\hline Otras & Mano \\
\hline Codo & Mediocarpiana \\
\hline $\begin{array}{l}\text { ¿Cuántas artroscopias se realizan en su centro/unidad } \\
\text { anualmente? }\end{array}$ & Otra \\
\hline ¿Se realizan artroscopias de revisión? & \multirow{2}{*}{$\begin{array}{l}\text { ¿Con qué casas comerciales trabaja habitualmente (marque } \\
\text { máximo 2)? }\end{array}$} \\
\hline \multirow{2}{*}{$\begin{array}{l}\text { ¿En qué \% aproximado del total realizan los siguientes } \\
\text { procedimientos? }\end{array}$} & \\
\hline & $\mathrm{S} \& \mathrm{~N}$ \\
\hline Fractura & Arthrex \\
\hline Artrolisis & Mitek \\
\hline Epicondilitis & Linvatec \\
\hline Cartílago/Cuerpos libres & Biomet \\
\hline Otra & Stryker \\
\hline \multirow{2}{*}{$\begin{array}{l}\text { ¿Con qué casas comerciales trabaja habitualmente (marque } \\
\text { máximo 2)? }\end{array}$} & Otras \\
\hline & Cadera \\
\hline S\&N & \multirow{2}{*}{$\begin{array}{l}\text { ¿Cuántas artroscopias se realizan en su centro/unidad } \\
\text { anualmente? }\end{array}$} \\
\hline Arthrex & \\
\hline Mitek & ¿Se realizan artroscopias de revisión? \\
\hline Linvatec & \multirow{2}{*}{$\begin{array}{l}\text { ¿En qué \% aproximado del total realizan los siguientes } \\
\text { procedimientos? }\end{array}$} \\
\hline Biomet & \\
\hline Stryker & Pinzamiento femoro acetabular \\
\hline Otras & Cartílago/cuerpos libres \\
\hline & Otra cirugía articular \\
\hline & Cirugía extrarticular \\
\hline & Otra \\
\hline
\end{tabular}




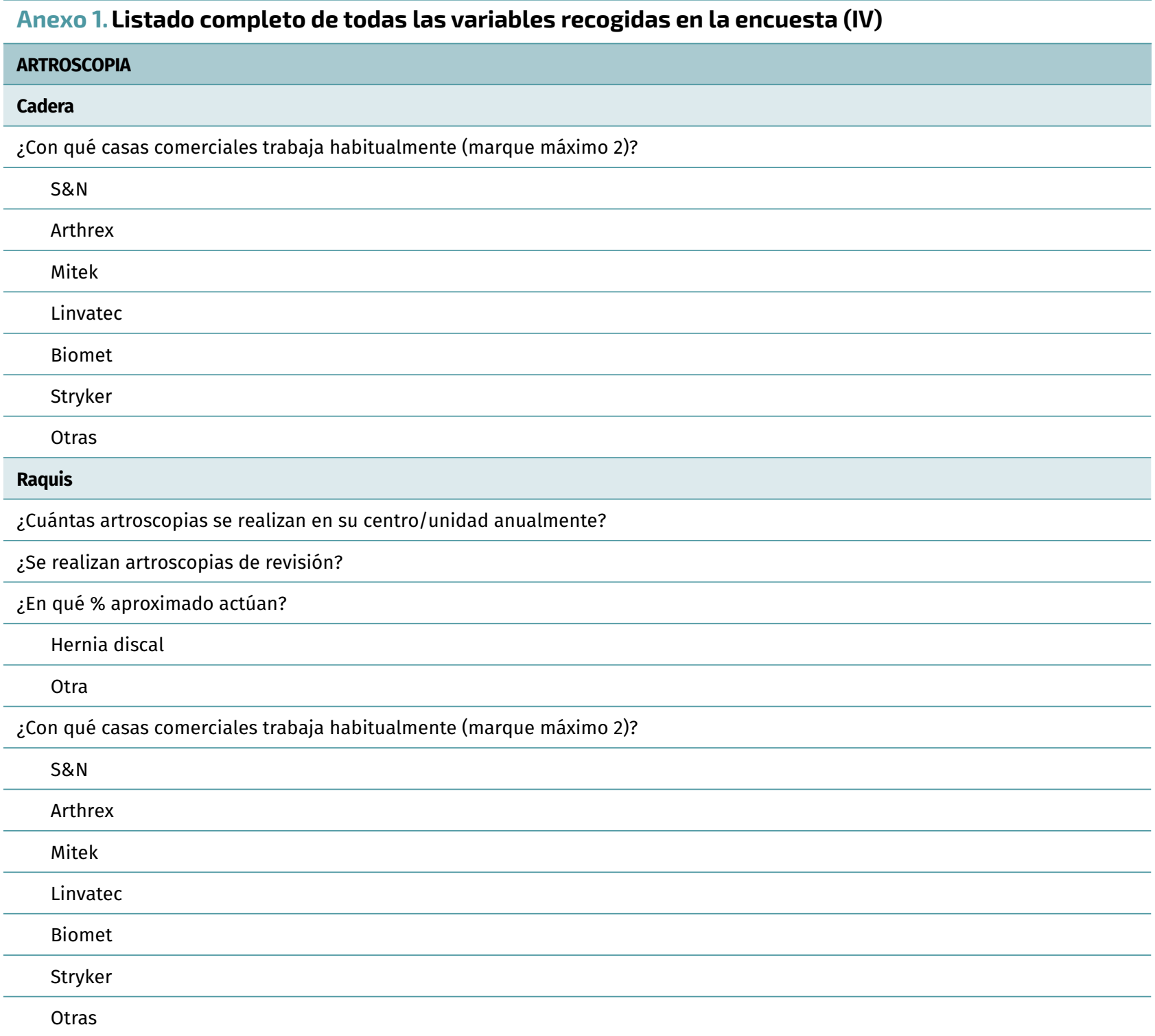




$$
35
$$

\section{Anexo 2. Capturas de pantalla de un ejemplo de encuesta rellena}
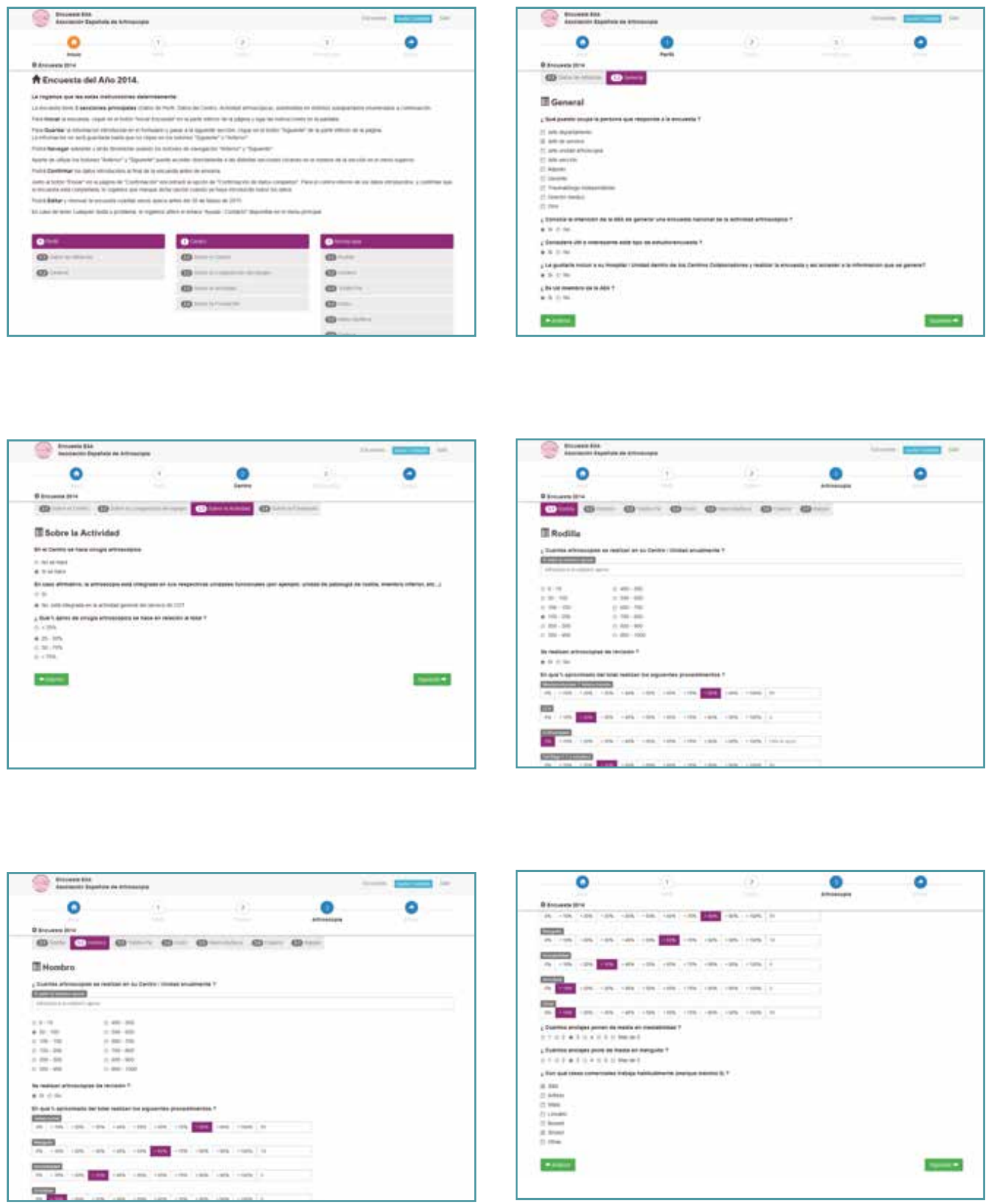